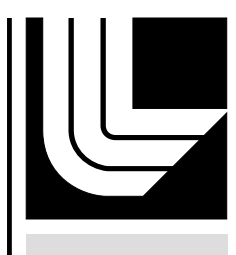

LAW RENCE LIVERMORE N A TIO N A L LABORATORY

\title{
UCRL-TR-219458
}

\section{Large-Scale Compton Imaging for Wide-Area Surveillance}

D. J. Lange, H. A. Manini, D. M. Wright

March 1, 2006 
This document was prepared as an account of work sponsored by an agency of the United States Government. Neither the United States Government nor the University of California nor any of their employees, makes any warranty, express or implied, or assumes any legal liability or responsibility for the accuracy, completeness, or usefulness of any information, apparatus, product, or process disclosed, or represents that its use would not infringe privately owned rights. Reference herein to any specific commercial product, process, or service by trade name, trademark, manufacturer, or otherwise, does not necessarily constitute or imply its endorsement, recommendation, or favoring by the United States Government or the University of California. The views and opinions of authors expressed herein do not necessarily state or reflect those of the United States Government or the University of California, and shall not be used for advertising or product endorsement purposes.

This work was performed under the auspices of the U.S. Department of Energy by University of California, Lawrence Livermore National Laboratory under Contract W-7405-Eng-48. 


\title{
Large-Scale Compton Imaging for Wide-Area Surveillance
}

\author{
David Lange, Hugh Manini, and Douglas Wright \\ High Energy Physics Group \\ N Division \\ Lawrence Livermore National Laboratory
}

February 24, 2006

\section{Introduction}

We study the performance of a large-scale Compton imaging detector placed in a low-flying aircraft, used to search wide areas for $\mathrm{rad} / \mathrm{nuc}$ threat sources. In this paper we investigate the performance potential of equipping aerial platforms with gamma-ray detectors that have photon sensitivity up to a few $\mathrm{MeV}$. We simulate the detector performance, and present receiver operating characteristics (ROC) curves for a benchmark scenario using a ${ }^{137} \mathrm{Cs}$ source. The analysis uses a realistic environmental background energy spectrum and includes air attenuation.

\section{Detection scenario}

The scenario we study here uses an unshielded ${ }^{137} \mathrm{Cs}(662 \mathrm{keV})$ source located somewhere on a large parking lot with a Compton imaging detector placed in an aircraft searching for the source from several hundred meters above. This is obviously an overly-simplified and relatively easy search scenario; however, it is useful for the sake of comparison to other systems and can be scaled to more realistic situations. 


\section{Background photon model}

We assume a total photon flux from the ground, integrated over the energy range of $90 \mathrm{keV}$ to $3 \mathrm{MeV}$, to be 2.2 counts $/ \mathrm{cm}^{2} / \mathrm{sec}$. This is at the high end of the range $\left(0.4-2.3\right.$ counts $\left./ \mathrm{cm}^{2} / \mathrm{sec}\right)$ that is observed for materials such as soil, concrete and gravel [1]. The photon energy spectra for these materials are crudely similar. For our study, we use an energy spectrum, shown in Figure 1, from a soil model calculated with

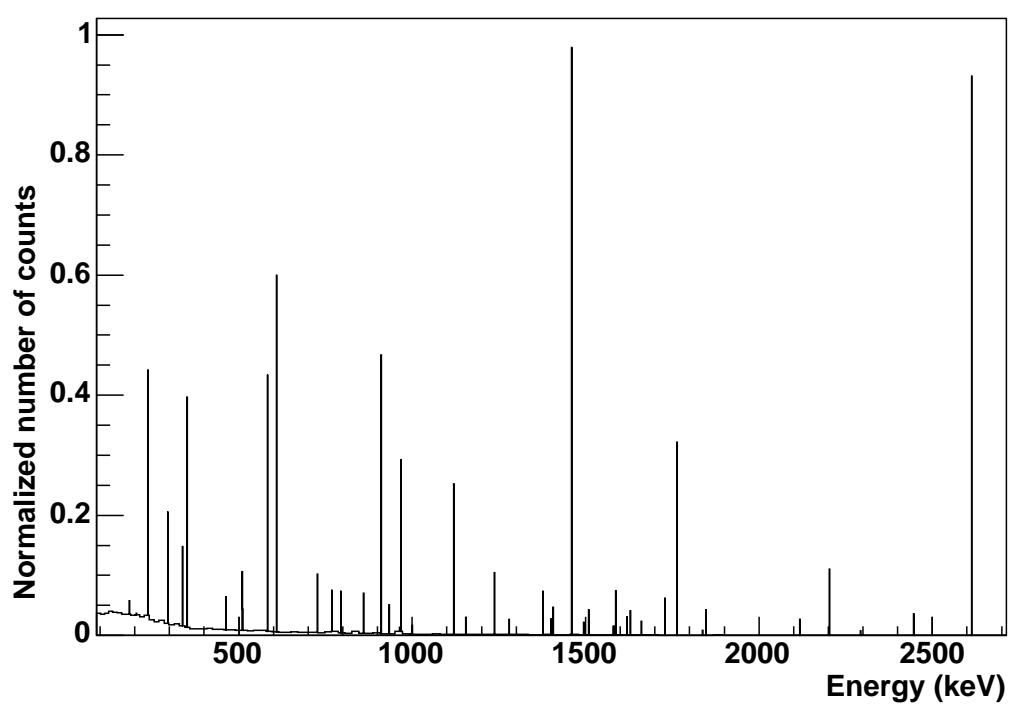

Figure 1: Calculated photon energy spectrum for dirt.

GAMGEN and MCNP from assumed isotopics for generic "dirt" [2]. Note that there is no $662 \mathrm{keV}$ line in the dirt spectrum, so the background is relatively low in the signal region of our scenario.

Ideally, we would model the production of background photons from a circular region of ground with a very large radius. This is computationally intensive in a full Monte Carlo simulation. The details of how we handle this are given in section 5.2. We have analytically calculated the background photon flux into the detector, to understand how the flux depends on the radius of the circular region of ground, and the detector height. Neglecting air attenuation and assuming that each patch of ground emits into $2 \pi$, one can integrate over a circular region beneath the detector to calculate the number of photons reaching the detector:

$$
N_{\gamma}=A_{d e t} B\left[1-\frac{1}{\sqrt{\left(\frac{r}{h}\right)^{2}+1}}\right],
$$

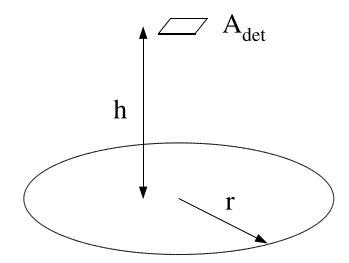

where $A_{d e t}$ is the area of a planar detector parallel to the ground, $B$ is the number of gamma rays per second per unit area emitted by the ground, $h$ is the height of the detector above the ground, and $r$ is the radius of the circular patch of ground directly below the detector. In the limit as $r$ goes to infinity, the equation above reduces to $N_{\gamma}=A_{d e t} B$. Note that a circular patch of ground with radius ten times the height $(r=10 h)$ contributes $90 \%$ of the total flux. 
The apparent solid angle subtended by the planar detector depends both on the distance away from the detector, and also on the angle from a point on the ground to the detector measured from the axis perpendicular to the detector area. This $\cos (\theta)$ effect is included in the formula for the planar detector. If instead of a planar detector one uses a spherical detector, then there is no $\cos (\theta)$ effect, and the number of photons reaching the spherical detector is:

$$
N_{\gamma}=A_{d e t} B \ln \left[\sqrt{\left(\frac{r}{h}\right)^{2}+1}\right]
$$

where $A_{d e t}$ is the cross-sectional area of the spherical detector, $B$ is the number of gamma rays per second per unit area emitted by the ground, $h$ is the height of the center of the spherical detector above the ground, and $r$ is the radius of the circular patch of ground directly below the detector. Note that the minimum possible value of $h$ is the radius of the spherical detector, not zero. For the spherical detector, in the limit as $r$ goes to infinity, the result for $N_{\gamma}$ goes to infinity. This is because there is no $\cos (\theta)$ effect to cause the projected detector area to approach zero along the direction of photon propagation for photons originating very far from the detector. The entire cross-sectional area of the spherical detector is the projected detector area for all directions.

\section{Detector angular resolution and the "ring effect"}

Angular resolution is a crucial parameter that determines the background-rejection power of the detector. Better resolution improves the sensitivity of the detector. A less obvious feature of ring-imaging type Compton detectors is that the random, environmental background contributes more than one would expect from simply scaling from the total $2 \pi$ background to the solid angle size of the signal region. This is a consequence of the degeneracy in the incident photon direction. There is a cone (which projects as a ring) of directions that kinematically fit the detected event. Since the background events are imaged as rings, not points, background events that are strictly outside the signal region produce rings which can still overlap the signal region. This "ring effect" is demonstrated below.

For this study, we have determined the angular resolution performance from simulations [3] of a hybrid silicon-germanium detector system with a $1 \mathrm{~m}^{2}$ active area, consisting of a $16 \times 16$ array of silicon-germanium detector modules. We used a monoenergetic $662 \mathrm{keV}$ point source located 100 meters away from the detector.

The signal region size is defined using the distance of closest approach (DOCA) between the reconstructed Compton rings and the true source location. In the case of a perfect detector with negligible Doppler broadening, each reconstructed ring will exactly intersect the source location (DOCA=0). Finite detector resolution (both in energy and position measurements) and the motion of the atomic electrons (Doppler effect) will broaden the DOCA distribution. Figure 2 shows the DOCA distribution from the simulation of a hybrid silicon-germanium detector system with a $1 \mathrm{~m}^{2}$ active area, for a $662 \mathrm{keV}$ point source and for "dirt" background. In Figure 2, the signal region has $85 \%$ of the signal within 5.5 degrees of the actual source location. In our analysis, we use a signal region radius of 5 degrees.

Although the signal region solid angle comprises only $0.4 \%$ of the $2 \pi$ field of view (looking downward only), the number of background rings which cross the signal region is significantly larger than $0.4 \%$ of the total number of background rings, due to the "ring effect". The simulations, as shown in Figure 2, predict that $8 \%$ (i.e. 20 times larger than $0.4 \%$ ) of the background rings overlap the signal region. 


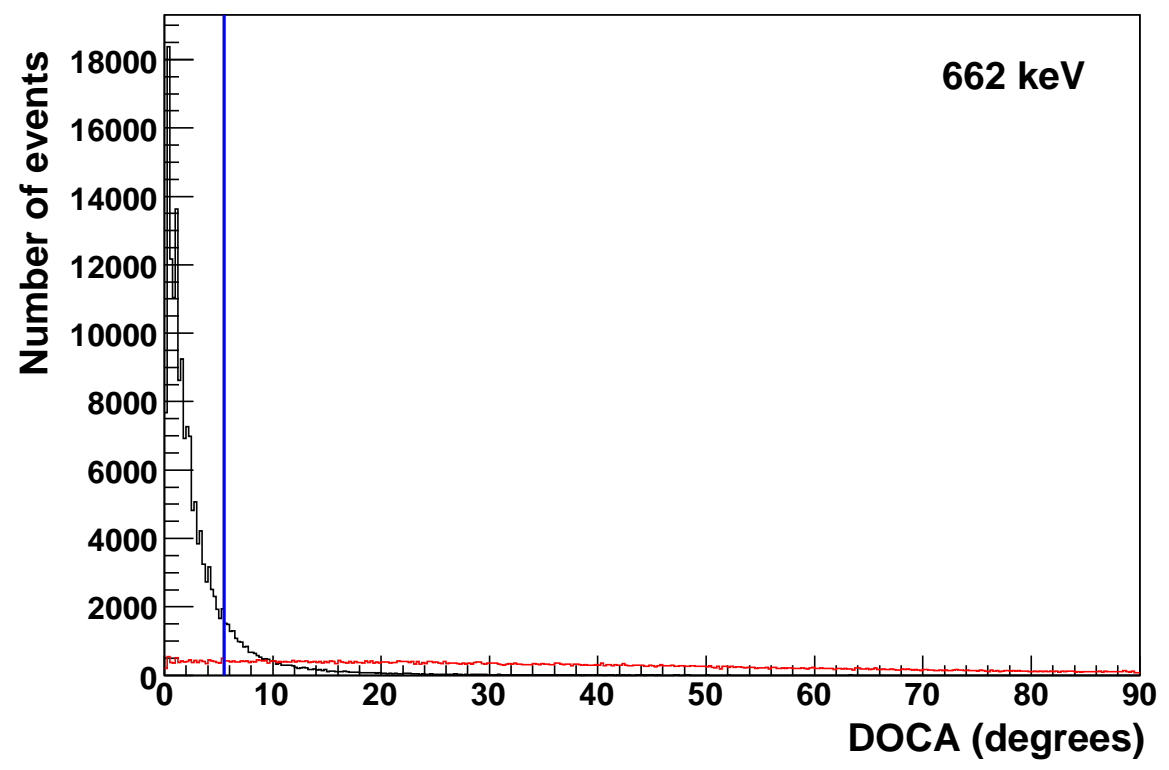

Figure 2: Simulated DOCA distributions for signal (black) and background (red), for a hybrid silicon-germanium detector system with an active area of $1 \mathrm{~m}^{2}$ for a $662 \mathrm{keV}$ point source with realistic detector parameters. The blue vertical line indicates the signal region boundary.

\section{Air attenuation}

\subsection{Signal contribution}

For photons from the threat source, we consider photons as signal only if they reach the detector unscattered. Any photon that is scattered will no longer give reliable directional information, nor will it pass an energycut requirement of $\pm 10 \mathrm{keV}$ when selecting specific spectral lines, which is often done to reduce background when searching for a specific source. The absorption length in air is between $80 \mathrm{~m}$ to $400 \mathrm{~m}$ for photons between $100 \mathrm{keV}$ and $10 \mathrm{MeV}$. To model the effects of air attenuation we utilize our full physics simulation using GEANT4 to track photons from a point source located some distance below the detector. We then determine the fraction of photons that reach the detector unscattered as a function of energy and a function of distance. These results are summarized in Figure 3. Below $500 \mathrm{keV}$, the effect of air attenuation is quite significant, even for short distances. For distances greater than $1 \mathrm{~km}$, less than $1 \%$ of photons at $3 \mathrm{MeV}$ will reach the detector unscattered.

\subsection{Background contribution}

Including the effects of air attenuation will further reduce the count rate of background photons originating far from the detector position. As with our analysis for air attenuation of the signal photons, we utilize our full GEANT4 simulation for the estimate of the background. Tracking photons through air from an infinite plane up to a finite detector is a very computationally intensive problem, since we need to include the ground area that extends at least ten times greater than the altitude, and since the probability for reaching the detector is low for typical photon energies and detector altitudes. To get around this computational problem, we run 

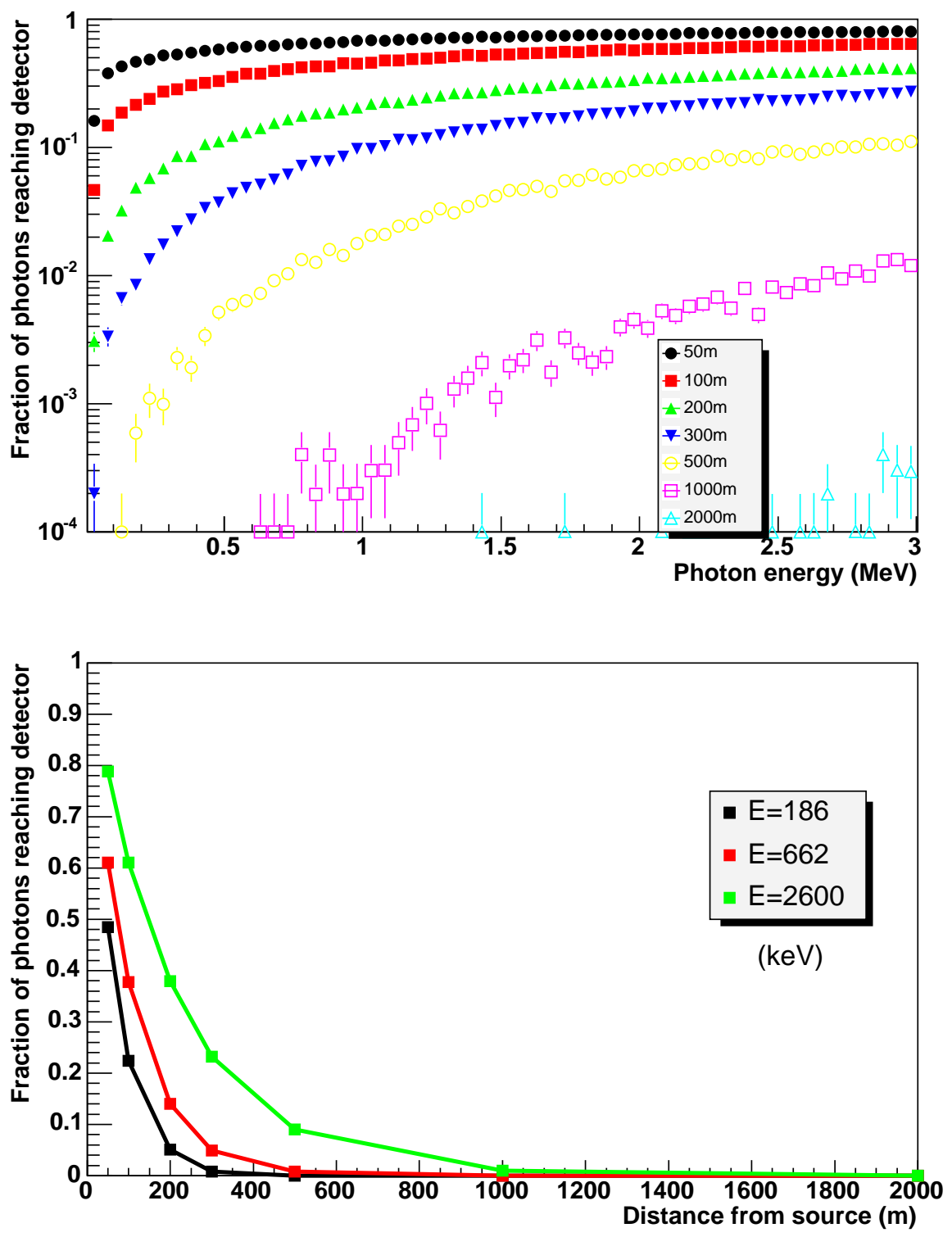

Figure 3: The fraction of photons from a point source that reach a detector unscattered by air, as a function of energy of the emitted photons, for various distances from the detector (top), and the fraction of photons from a point source that reach a detector unscattered by air, as a function of the distance from the source to the detector, for a few specific energies in units of keV (bottom). All emitted photons are generated so that they will strike the detector if they are not scattered before reaching it. 
the simulation using the background energy spectrum model described previously, generating photons from a single point. These photons are tracked through air and we tally the photon energy and location as it crosses particular altitudes, considering only the upward crossing (we assume we will shield anything from above). We ignore any contribution from other particles, such as electrons, generated by interactions in the air. We consider intersections out to a radius of $3 \mathrm{~km}$ and we choose various detector altitudes, including $10 \mathrm{~m}, 100 \mathrm{~m}$, and $300 \mathrm{~m}$. At the end of this simulation, we have triplets of altitude, observed photon energy, and observed radius for a large number of generated photons. Where photons cross a particular altitude multiple times, we give each crossing a weight of $1 / N$ to ensure that a photon can interact with a detector at most once (at one of the $N$ radii).

To compute the total background observed by a detector at a particular altitude, we select triplets with the specified altitude and create a matrix of energy vs. radius. This information can then be used to derive an energy spectrum observed for a detector at this altitude due to a semi-infinite background plane. Table 1 and Figure 4 show the final background counts for several detector altitudes that we considered, as well as the variation in the importance of photon contributions with a given $r$ and $E$ as the detector altitude is changed.

\begin{tabular}{ccc}
$\begin{array}{c}\text { Altitude } \\
(\mathrm{m})\end{array}$ & $\begin{array}{c}0.1-10 \mathrm{MeV} \\
\left(\text { counts } / \mathrm{cm}^{2} / \mathrm{sec}\right)\end{array}$ & $\begin{array}{c}662 \pm 10 \mathrm{keV} \\
\left(\text { counts } / \mathrm{cm}^{2} / \mathrm{sec}\right)\end{array}$ \\
\hline 0 & 2.2 & 0.013 \\
10 & 1.73 & 0.011 \\
100 & 0.99 & 0.006 \\
300 & 0.29 & 0.002 \\
\hline
\end{tabular}

Table 1: Count rate of photons reaching the detector as a function of detector altitude for the full energy range $(0.1-10 \mathrm{MeV})$ and $662 \pm 10 \mathrm{keV}$.

\section{Receiver Operating Characteristics (ROC) curves}

Using the scenario and physics inputs described above, we evaluate the detector performance using analytic calculations and quote results using ROC curves to characterize the performance of a detector in a particular configuration with respect to a source. ROC curves show the probability of signal detection versus the probability of false signal detection. We convert the probability of false signal detection into a false-alarm rate in our ROC curves, using the total measurement time.

We have confirmed the accuracy of the analytic ROC curve calculations by comparing them with a Monte Carlo method that simulates the outcome of numerous experiments. In other words, we generate results from individual experiments by sampling the parent distributions. We then count the signal and background in each experiment and determine if there is a false alarm in any imaging bin, just like the analysis of real data. We can then build up a ROC curve by simulating many experiments. This Monte Carlo method is much more computationally intensive than the analytic calculations, and the Monte Carlo method requires high statistics to accurately measure the region of the ROC curve that has low false-alarm rates. We find that the analytic calculations are in excellent agreement with the Monte Carlo method. Not only are the analytic calculations faster, but they also allow us to make very accurate estimates of the detector performance for very low false-alarm rates, which is of course the most desirable region for real concepts of operations (CONOPS). 

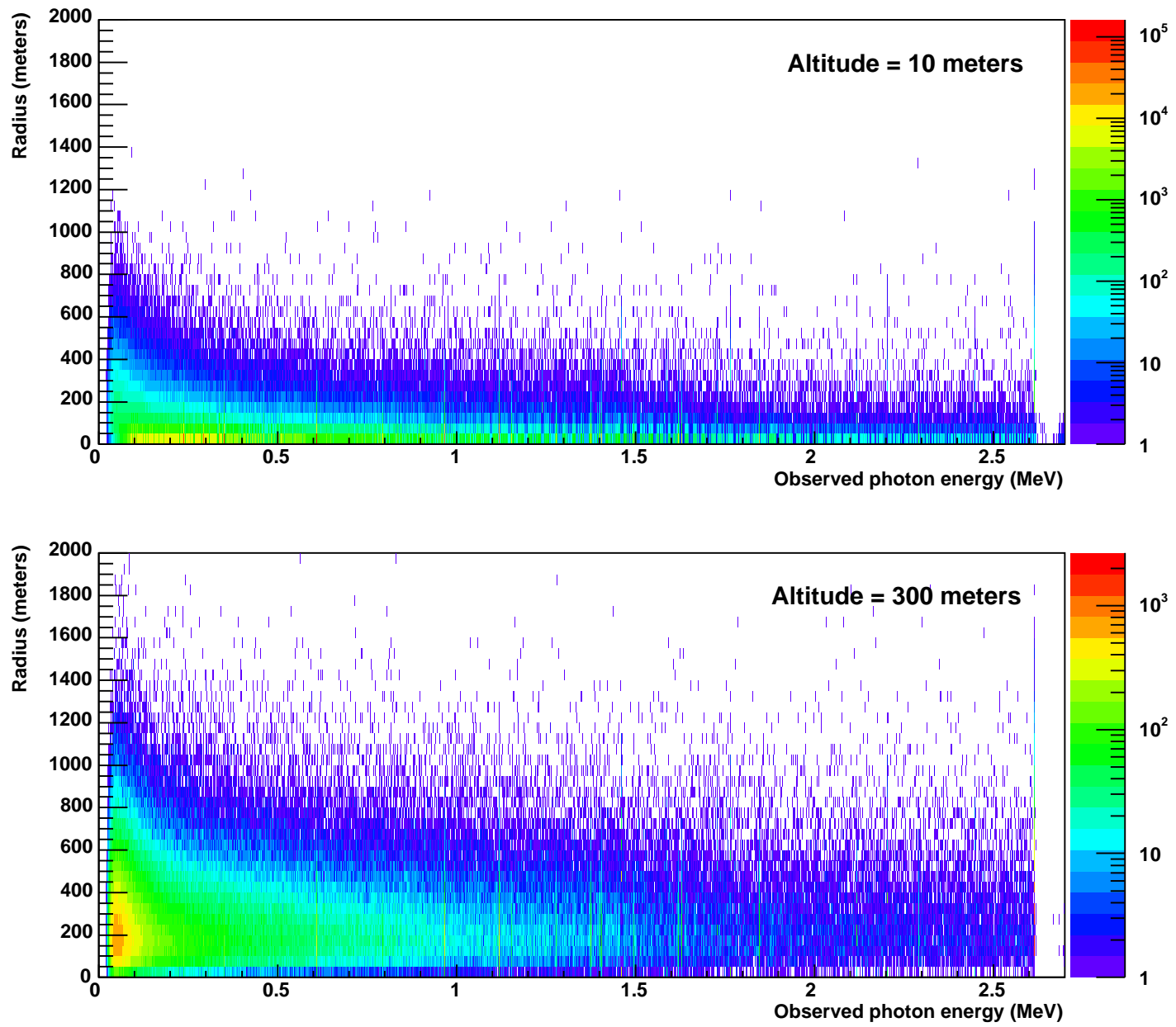

Figure 4: Distributions of the observed background photon propagation radius versus observed energy, for altitudes of $10 \mathrm{~m}$ (top) and $300 \mathrm{~m}$ (bottom). The color scale is a log scale for the number of background counts. 
Our analytic ROC curve calculations take the following as input:

1. Measurement time.

2. Detector area (nominally $1 \mathrm{~m}^{2}$ ).

3. Detector orientation (detector is planar, so it can point in different directions).

4. Detector altitude.

5. Detector efficiency.

6. Source emission rate.

7. Air attenuation of signal photons.

8. Background photon flux into the detector, from either the full 90-3000 keV energy spectrum or within a $\pm 10 \mathrm{keV}$ energy-cut window. Effects of air attenuation and scattering are included. The background is assumed to come randomly from $2 \pi$ (i.e. from the ground only).

9. Signal region size (resolution).

10. The number of background rings which cross the signal region ("ring effect").

We calculate our ROC curves analytically using Poisson probability distributions for the detected count rate of background and source plus background. We assume that the signal region, which contains $85 \%$ of the total signal contribution, can be isolated to a localized region of solid angle. We set the imaging bin size to correspond to this signal region solid angle. Since the background photons are imaged as rings, not points, the number of background rings seen in one imaging bin is much greater than the total number of background counts divided by the total number of imaging bins. According to our simulations, a typical signal region occupies $1 / 250$ of the $2 \pi$ solid angle, however $20 / 250$ of the background rings cross the signal region. Thus, this "ring effect" causes the Compton imaging detector to see about 20 times more background in the signal region than it would if it detected background events as points which were uniformly distributed throughout $2 \pi$. We divide the $2 \pi$ image solid angle into 250 imaging bins, and the total signal occupies one imaging bin. Each of the 250 imaging bins contains background, so every imaging bin has the possibility of producing a false alarm.

In constructing a ROC curve, we must chose a detector threshold above which a false alarm is generated. During the measurement time, if the number of background counts fluctuates above the detection threshold, then the background fluctuation looks like a source (generating a false alarm). In our method we use true Poisson distributions for the source and background distributions, so that we correctly model situations with low counting statistics.

Figure 5 shows example Poisson distributions of background and signal plus background. We numerically integrate the Poisson distributions to determine the detection and false detection probabilities. We have also checked that our numerical integration gives the correct result for both high statistics situations (when a Gaussian approximation could be used) and low statistics situations (when the Gaussian approximation is wrong).

The entire ROC curve is mapped out by varying the detection threshold. Note that for an imaging detector, a false alarm can come from any of the spatial imaging bins, so we report the false-alarm rate as the total number of alarms per unit time. Thus, making smaller bins will tend to increase the false-alarm rate because there will be more bins with low count rates and thus more chances for the background to fluctuate above the detection threshold. 


\section{Poisson Distributions}

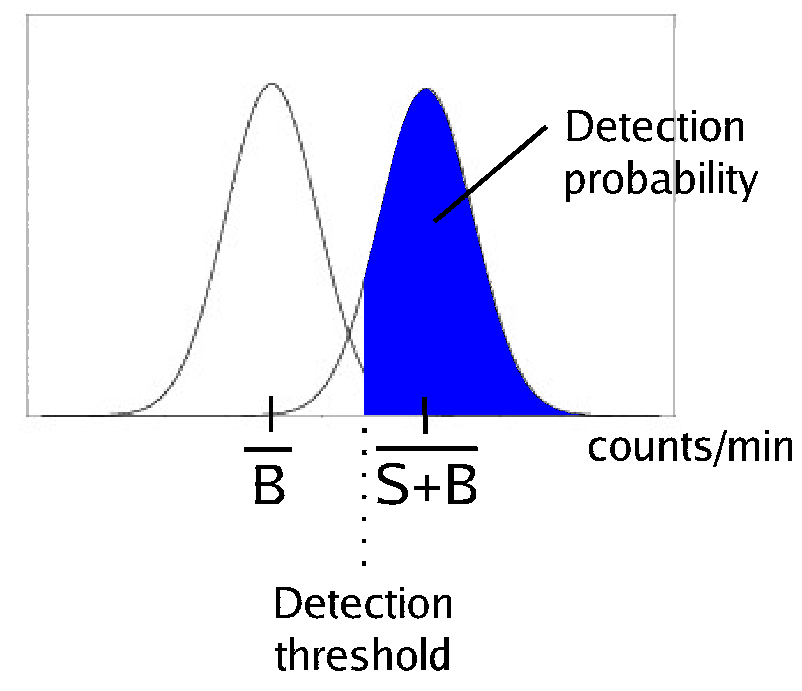

\section{Poisson Distributions}

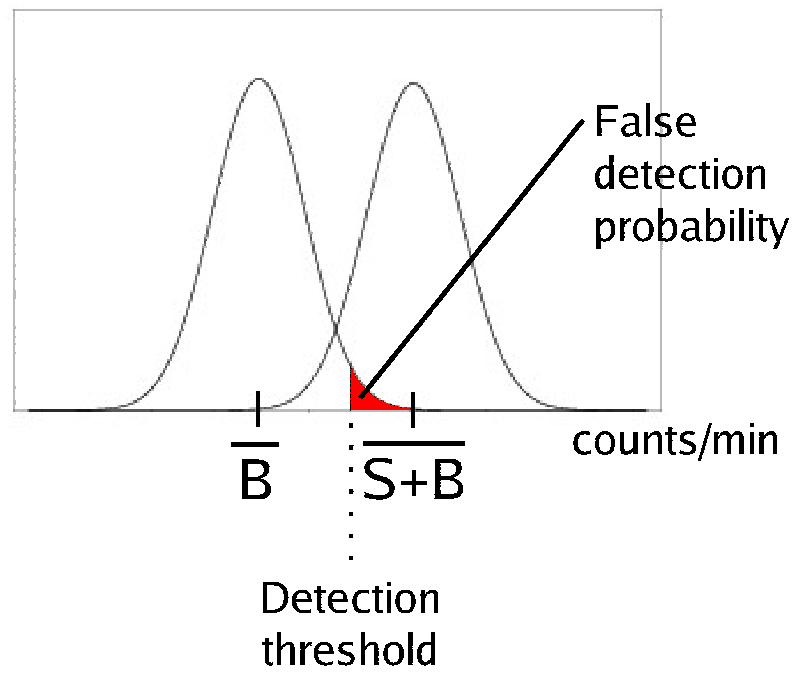

Figure 5: Poisson probability distributions for background and source plus background, showing the detection probability (top) and the false detection probability (bottom), for a particular value of the detection threshold. 


\section{Results}

Figure 6 shows ROC curves for a ${ }^{137} \mathrm{Cs}$ source located $100 \mathrm{~m}$ directly below a $1 \mathrm{~m}^{2}$ detector, with a measurement time of 100 seconds, a detector efficiency of $100 \%$, and an energy-cut window of $662 \pm 10 \mathrm{keV}$. Note that the energy-cut window reduces the background significantly. We varied the source strength until we found the source strength value of $0.007 \mathrm{mCi}$ at which a ROC curve passes through approximately $50 \%$ detection probability at one false alarm per hour. We find that for very small false-alarm rates, small changes in the source strength can produce significant changes in the detection probability. For example, in Figure 6 we illustrate that doubling the source strength to $0.014 \mathrm{mCi}$ makes the source easily observed in the same scenario with a high detection probability and a very small false-alarm rate.

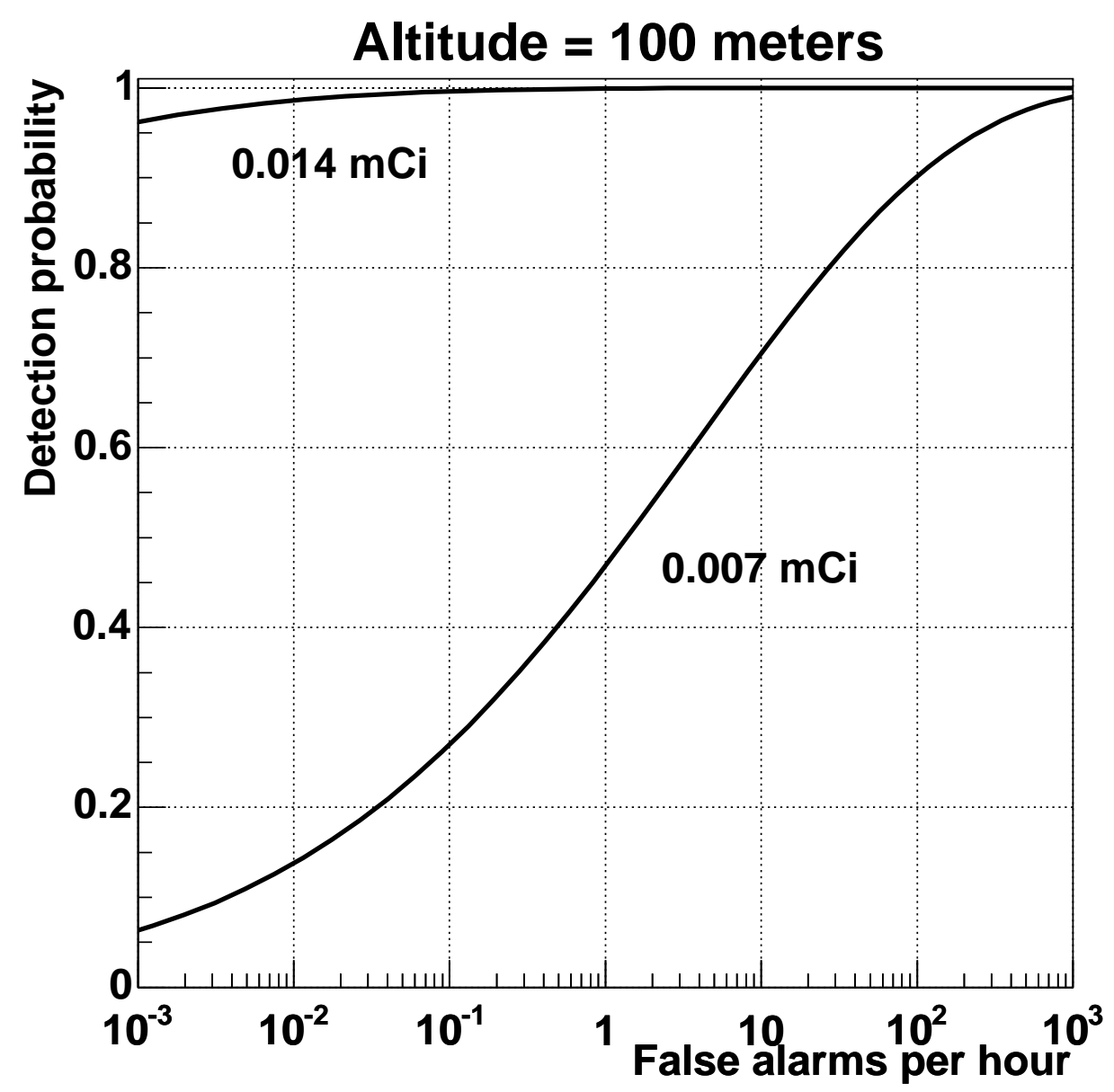

Figure 6: ROC curves for a ${ }^{137} \mathrm{Cs}$ source with an energy-cut window of $662 \pm 10 \mathrm{keV}$. 
Figure 7 shows the measurement time versus observed source strength for a $1 \mathrm{~m}^{2}$ detector at altitudes of $100 \mathrm{~m}$ and $300 \mathrm{~m}$, for two different detection probabilities, with one false alarm per hour, with $100 \%$ detector efficiency, with an energy-cut window of $662 \pm 10 \mathrm{keV}$, and with the source located at the point on the ground directly below the detector. Figure 7 shows that for one false alarm per hour, requiring a detection probability of $95 \%$ instead of $50 \%$ can have a significant effect on the required measurement time. For example, for a source strength of $0.01 \mathrm{mCi}$, the measurement time for $50 \%$ detection probability is about 50 seconds, while the measurement time for $95 \%$ detection probability is about 100 seconds.

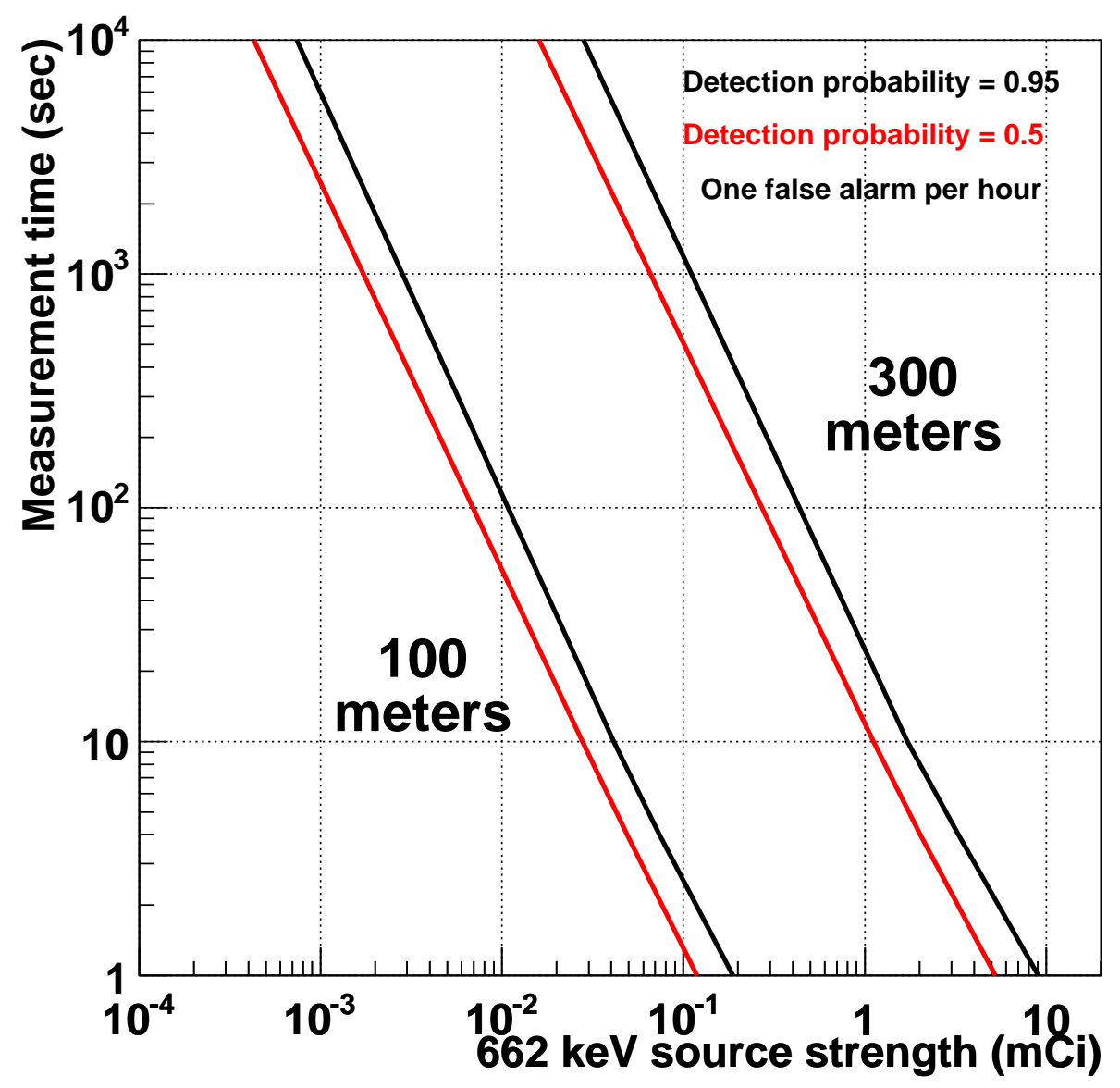

Figure 7: Measurement time versus source strength, for a detection probability of $95 \%$ (black), and for a detection probability of $50 \%$ (red). 
Figure 8 shows the measurement time versus observed source strength for a $1 \mathrm{~m}^{2}$ detector at altitudes of $50 \mathrm{~m}, 100 \mathrm{~m}, 300 \mathrm{~m}$, and $500 \mathrm{~m}$, with a source detection probability of $95 \%$, with one false alarm per hour, with $100 \%$ detector efficiency, with an energy-cut window of $662 \pm 10 \mathrm{keV}$, and with the source located at the point on the ground directly below the detector. Figure 8 shows that a practical upper limit to the altitude of the detector is about $300 \mathrm{~m}$, for a $1 \mathrm{mCi}^{137} \mathrm{Cs}$ source. For example, at an altitude of $300 \mathrm{~m}$, a $1 \mathrm{mCi}{ }^{137} \mathrm{Cs}$ source can be detected with a measurement time of about 25 seconds; however, at an altitude of $500 \mathrm{~m}$, in a measurement time of about 25 seconds, a $10 \mathrm{mCi}^{137} \mathrm{Cs}$ source can be detected. The separation along the source-strength axis between the $100 \mathrm{~m}$ and $300 \mathrm{~m}$ results is larger than the separation between the $300 \mathrm{~m}$ and $500 \mathrm{~m}$ results, because the detector solid angle decreases by $89 \%$ from altitudes of $100 \mathrm{~m}$ to $300 \mathrm{~m}$, but only by $64 \%$ from $300 \mathrm{~m}$ to $500 \mathrm{~m}$. Also, the air attenuation causes the source photon flux to decrease by $87 \%$ between $100 \mathrm{~m}$ and $300 \mathrm{~m}$, but only by $84 \%$ between $300 \mathrm{~m}$ and $500 \mathrm{~m}$.

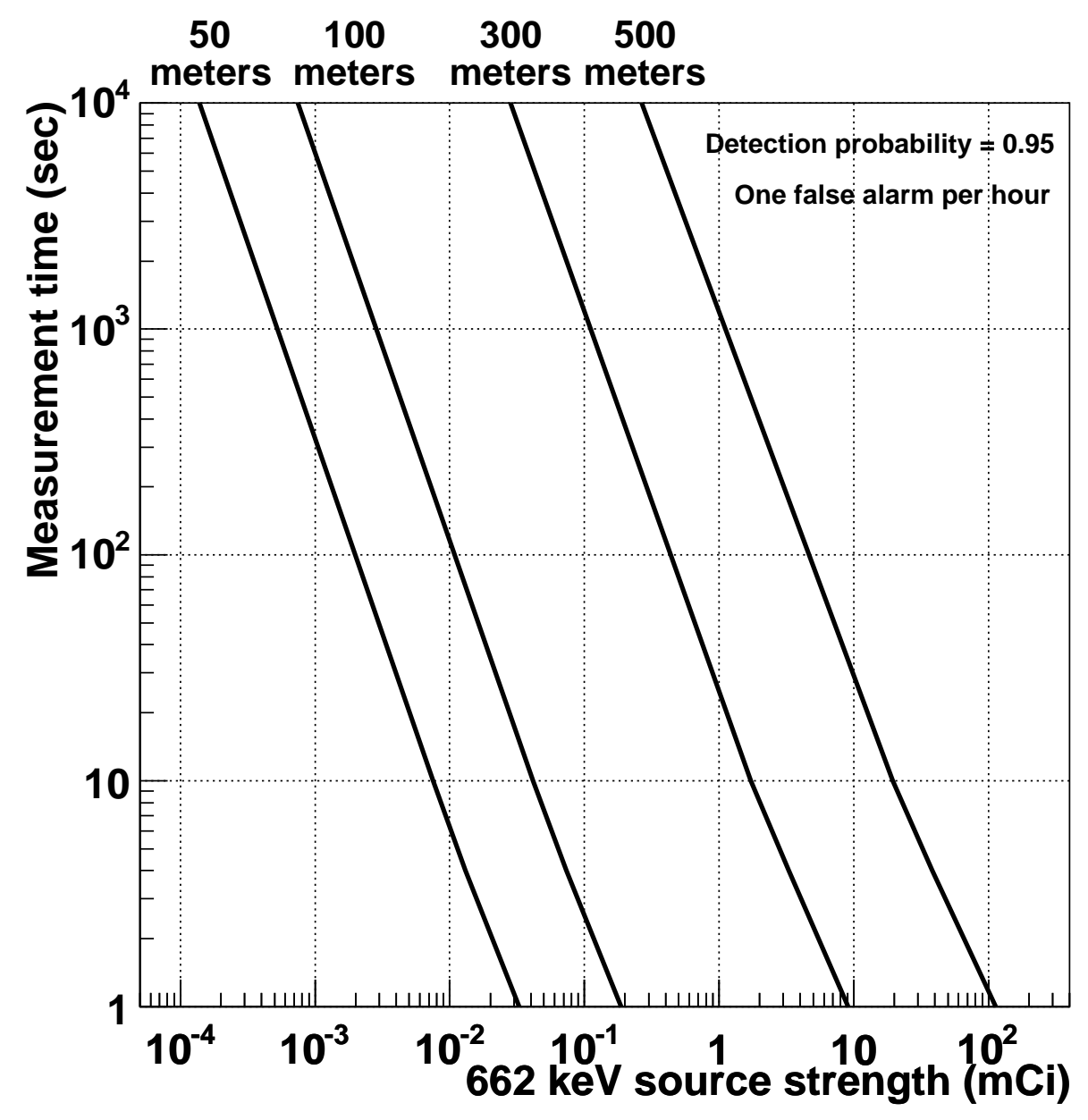

Figure 8: Measurement time versus source strength. 
Figure 9 shows the measurement time versus the distance along the ground from the source to the point directly below the detector, for a $1 \mathrm{~m}^{2}$ detector, for altitudes of $100 \mathrm{~m}$ and $300 \mathrm{~m}$, for two different detector configurations, for an observed source strength of $1 \mathrm{mCi}$, a detection probability of $95 \%$, with one false alarm per hour, with a detector efficiency of $100 \%$, and with an energy-cut window of $662 \pm 10 \mathrm{keV}$. For large distances along the ground, the detector solid angle, from the position of the source, becomes comparable for the two cases of the $100 \mathrm{~m}$ altitude and the $300 \mathrm{~m}$ altitude. However, at $662 \mathrm{keV}$, the background count rate is three times greater at an altitude of $100 \mathrm{~m}$ than it is at an altitude of $300 \mathrm{~m}$. Thus, for large distances along the ground, it becomes advantageous to position the detector at an altitude of $300 \mathrm{~m}$ rather than at an altitude of $100 \mathrm{~m}$.

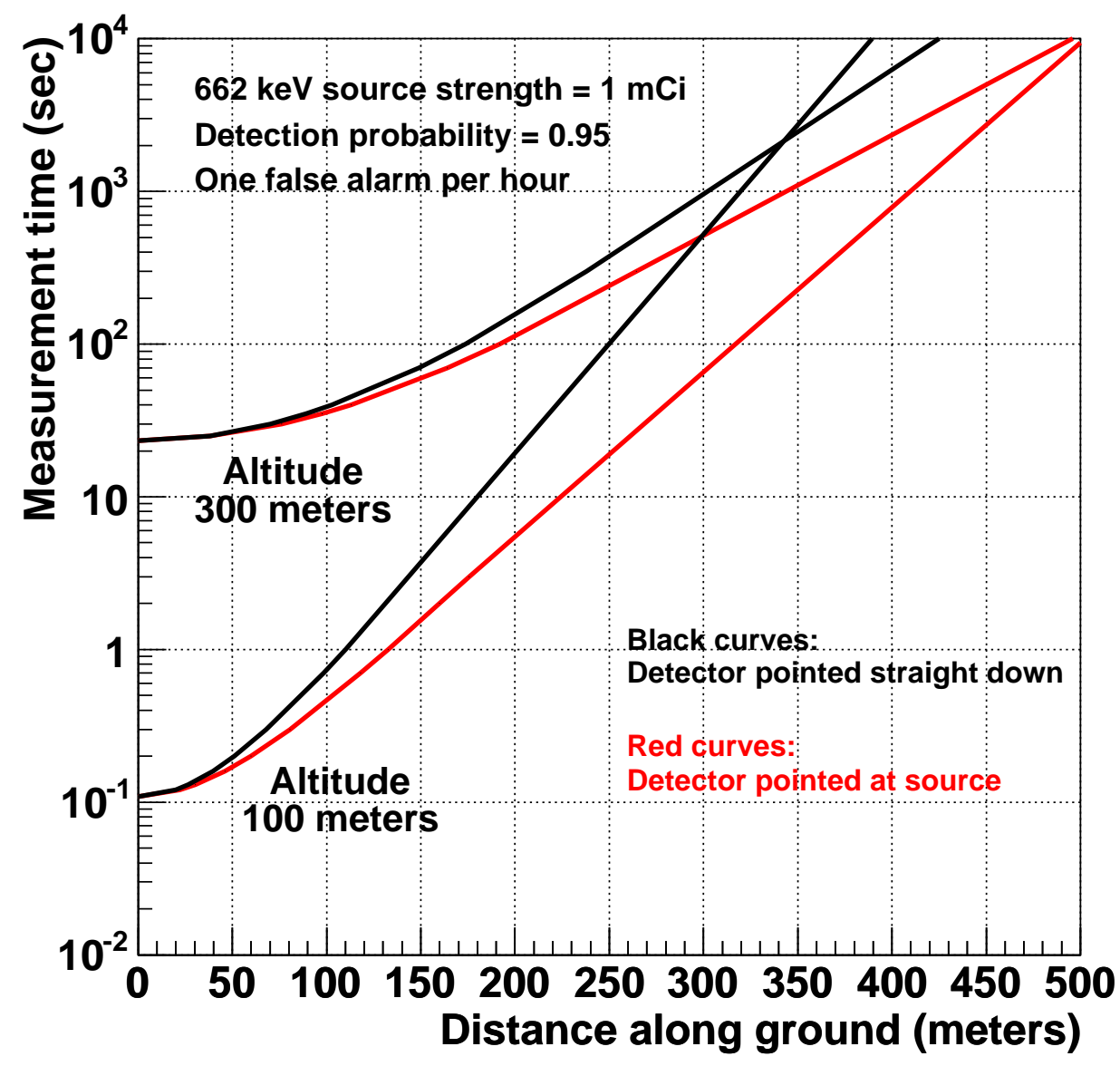

Figure 9: Measurement time versus distance of the source along the ground, for the detector pointed straight down (black), and for the detector pointed at the source (red). 
Figure 10 shows the measurement time versus detector efficiency for a ${ }^{137} \mathrm{Cs}$ source, for a $1 \mathrm{~m}^{2}$ detector at an altitude of $100 \mathrm{~m}$, a detection probability of $95 \%$, with one false alarm per hour, with an energy-cut window of $662 \pm 10 \mathrm{keV}$, with the source located at the point on the ground directly below the detector. Figure 10 shows that doubling the detector efficiency reduces the required measurement time by about a factor of two.

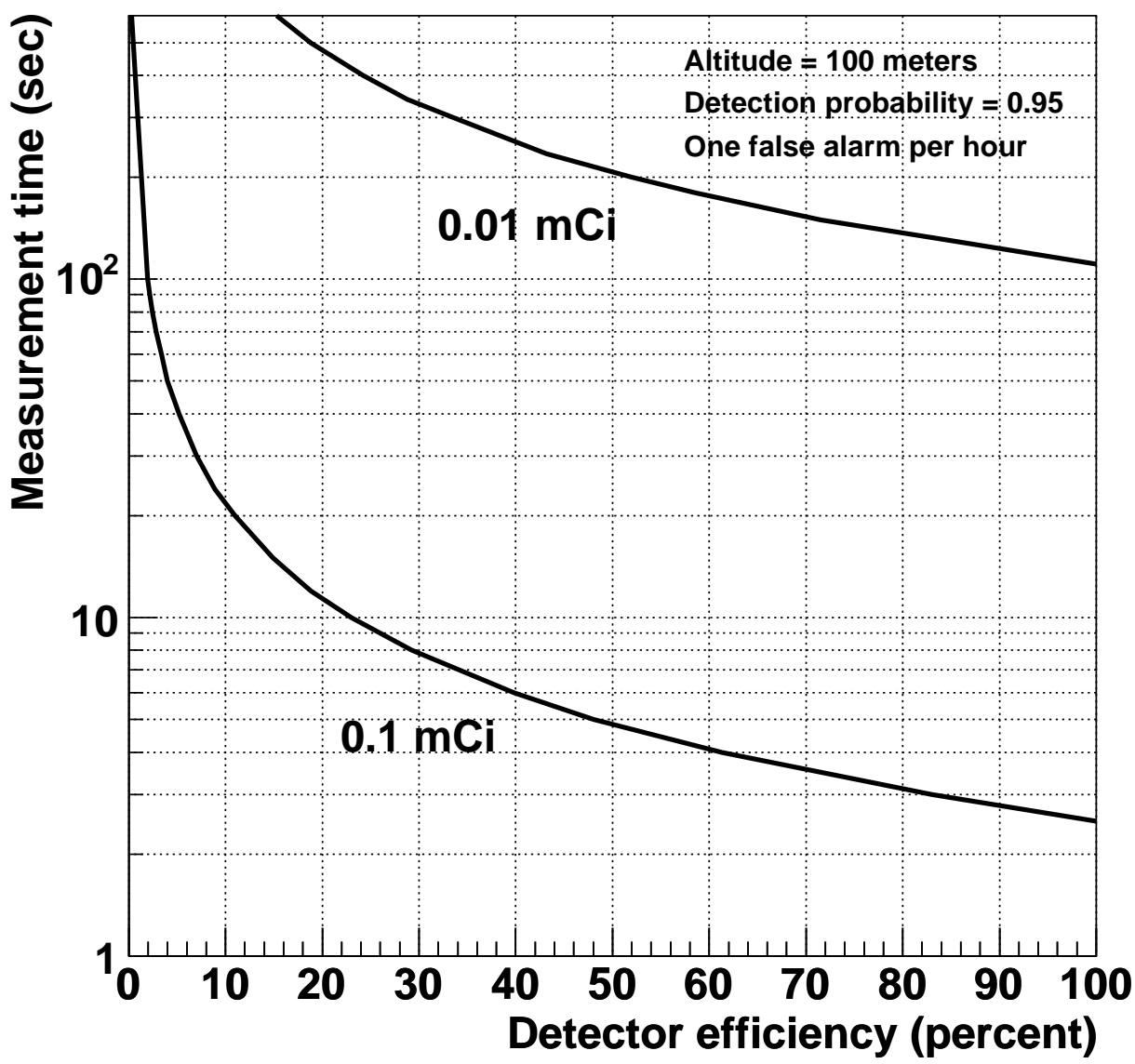

Figure 10: Measurement time versus detector efficiency.

\section{References}

[1] L. E. Smith, et al., An Evaluation Framework for Search Instruments.

[2] J. Gronberg, S. Johnson, D. Lange, D. Wright, Source and Background Gamma-ray Spectra, UCRL-TR-201636.

[3] D. Lange, H. Manini, D. Wright, Simulation and Analysis of Large-Scale Compton Imaging Detectors, UCRL-TR-. 